\title{
Unique Aspects of Cryptochrome in Chronobiology and Metabolism, Pancreatic $\beta$-Cell Dysfunction, and Regeneration: Research into Cysteine414-Alanine Mutant CRY1
}

\begin{abstract}
Satoshi Okano
Research Center for Molecular Genetics, Institute for Promotion of Medical Science Research, Yamagata University Faculty of Medicine, Yamagata 990-9585, Japan

Correspondence should be addressed to Satoshi Okano; sokano@med.id.yamagata-u.ac.jp

Received 6 November 2016; Accepted 27 November 2016

Academic Editor: Hiroshi Okamoto

Copyright (C) 2016 Satoshi Okano. This is an open access article distributed under the Creative Commons Attribution License, which permits unrestricted use, distribution, and reproduction in any medium, provided the original work is properly cited.

Cryptochrome proteins (CRYs), which can bind noncovalently to cofactor (chromophore) flavin adenine dinucleotide (FAD), occur widely among organisms. CRYs play indispensable roles in the generation of circadian rhythm in mammals. Transgenic mice (Tg mice), ubiquitously expressing mouse CRY1 having a mutation in which cysteine414 (the zinc-binding site of CRY1) being replaced with alanine, display unique phenotypes in their circadian rhythms. Moreover, male Tg mice exhibit symptoms of diabetes characterized by beta-cell dysfunction, resembling human maturity onset diabetes of the young (MODY). The lowered proliferation of $\beta$-cells is a primary cause of age-dependent $\beta$-cell loss. Furthermore, unusually enlarged duct-like structures developed prominently in the Tg mice pancreases. The duct-like structures contained insulin-positive cells, suggesting neogenesis of $\beta$-cells in the Tg mice. This review, based mainly on the author's investigation of the unique features of Tg mice, presents reported results and recent findings related to molecular processes associated with mammalian cryptochromes, especially their involvement in the regulation of metabolism. New information is described with emphasis on the aspects of islet architecture, pancreatic $\beta$-cell dysfunction, and regeneration.
\end{abstract}

\section{Various Functions of Cryptochromes in Animals}

Before addressing the main subject, one can concisely outline the diverse roles of cryptochromes in animals along with the track of related research. Cryptochrome proteins, which can bind noncovalently to cofactor (chromophore) flavin adenine dinucleotide (FAD), occur widely among organisms [1]. The term cryptochrome itself was coined around 1970 to describe hitherto unknown photoreceptors in plants: crypto means hidden or mysterious in Greek. The cryptochromes are evolutionally conserved and are structurally related to photolyase, a DNA repair enzyme [1]. Figure 1 portrays a phylogenetic tree of photolyase/cryptochrome family proteins referred to in the literature [2]. Efforts undertaken for more than half century to elucidate the molecular processes of DNA repair began with the discovery of photolyase and culminated in the awarding of a Nobel Prize in Chemistry in 2015 [3].
Cryptochromes also play fundamentally important roles in the circadian clock of organisms living on earth, enabling them to adapt to daily $24 \mathrm{~h}$ cycles.

Mammals exhibit various physiological and behavioral processes that show rhythms: sleep/wake activity, secretion of hormones, and metabolism fluctuate rhythmically in approximately $24 \mathrm{hr}$ cycles. These phenomena are regulated by an internal circadian clock in the body of each individual [4]. Already by the 1970s, the central site of the mammalian circadian clock was singled out anatomically as the suprachiasmatic nucleus (SCN) [5-7], but the molecular clockwork in the SCN remained elusive until the last few years of the 20th century [4]. Along with the advance of molecular biology and genetics, the long sought-after molecular mechanism of the mammalian circadian rhythm was discovered during a short period in the late 1990s [4]. The clock genes were cloned during that period [4]. Mammalian cryptochromes 1 and 2 (Cry1 and Cry2) were also identified and characterized 


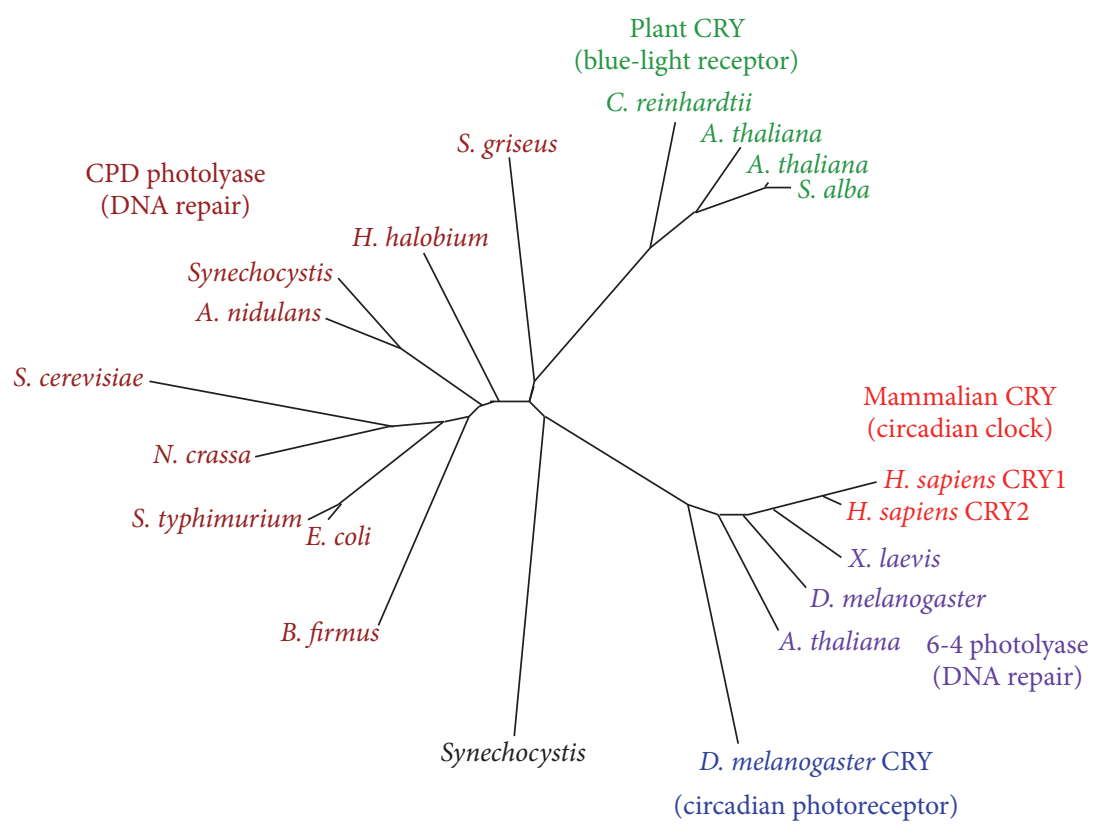

FIgURE 1: Phylogenetic tree of photolyase/cryptochrome family proteins (adapted from [2]).

during that time [8-11]. A series of knockout (KO) studies in mice played crucial roles in determining the function of CRYs in mammals: the Yasui group produced mutants that were defective in each of Cry1 or Cry 2 or in both genes. This research group, in conjunction with Van Der Horst et al., found that the mutants exhibited no rhythmic behavior when kept in constant darkness [12]. Independently, using a similar double knockout strategy, another group reached fundamentally identical conclusions [13]. The result, along with those of an in vitro study [14], proved that CRYs are indispensable components of clock proteins. Single Cry1 KO mice show a shorter period phenotype but single Cry2 KO mice present a longer period phenotype than that of wildtype control mice [12,13]. These results suggest that the functions played by these CRYs in the regulation of circadian clock are not the same.

By 2000, the basic molecular mechanism of mammalian circadian rhythm was identified as a cyclic transcriptional/translational feedback loop (TTFL), which comprises four classes of proteins: Periods (PERs), cryptochromes (CRYs), CLOCK, and BMAL1 [4]. A schematic diagram of mammalian TTFL is presented in Figure 2. In the feedback loop, CRY proteins form dimers with PER proteins in the cytoplasm. Thereafter, they enter the nucleus and play the role of a transcriptional repressor to the CLOCK/BMAL1 complex [4] (Figure 2). Nuclear receptor family proteins of two kinds, REV-ERBs and RORs, form another loop of the molecular clock $[4,16]$. The second molecular loop plays a role in stabilizing TTFL [16]. Regarding Drosophila melanogaster, the Drosophila Period gene was cloned originally long before in 1984, but the molecular mechanism of the circadian rhythm was elucidated only during the last few years of the 20th century, almost simultaneously with the mammalian circadian rhythm [17]. A negative feedback

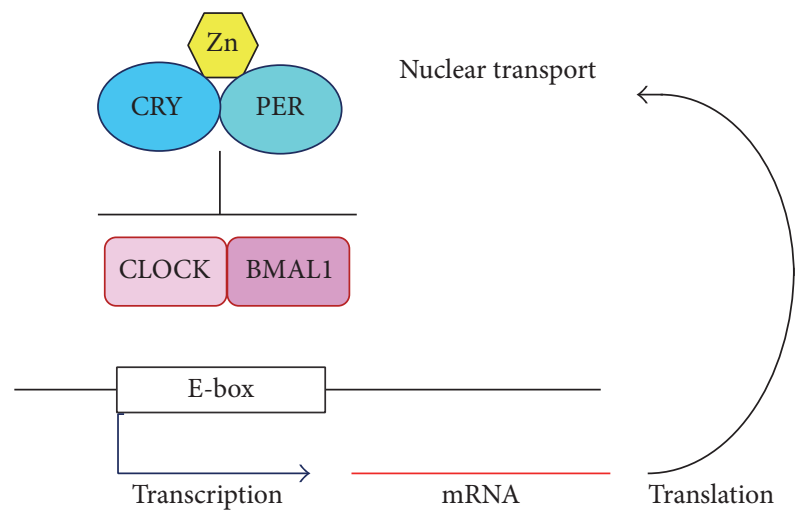

FIGURE 2: Molecular transcriptional/translational feedback loop (TTFL) model for mammalian circadian clock.

loop similar to that of TTFL in mammals was identified comprising four proteins: Period, Timeless, CLOCK, and Cycle [17]. The main role of Drosophila CRY turned out to be that of a photoreceptor $[1,17,18]$. Still another aspect of cryptochromes that is recently attracting many researchers' interest is the function of "magnetoreception." For instance, coherent quantum mechanical analyses [19] predicted that photoexcitation of cryptochrome proteins in the avian retina generated radical pairs (pairs of electron spins), which enable migratory birds to detect the direction of earth's weak magnetic field. Results of that study suggest that the radical pair magnetoreception in cryptochrome can be regarded as a quantum biology phenomenon [19].

Mammalian CRYs are subject to various modifications posttranslationally, as described below. Such multilateral regulations of CRYs are believed to regulate or fine-tune the 
oscillation of mammalian molecular clocks. Degradation of CRY proteins is conducted via the ubiquitin system. AMPactivated protein kinase (AMPK), which is known to work as a metabolic sensor, phosphorylates CRY proteins [20] and regulates CRY protein stability by leading CRY proteins to bind to a ubiquitin ligase: FBXL3 [21-23]. FBXL21, another ubiquitin ligase, stabilizes CRY proteins [24, 25]. Ubiquitinspecific protease 7 (USP7) deubiquitinates CRYs and thereby stabilizes CRYs [26]. Furthermore, posttranscriptional regulation has been reported to occur by hnRNP Q, an RNAbinding protein, which controls translation of Cryl mRNA [27].

\section{Structural-Biological Analyses of CRYs}

Recent X-ray crystallographic analysis conducted along with investigations of molecular biology has provided important clues that have elucidated the molecular roles of CRYs. Structural studies have elicited details of interaction of CRYs with FBXL3 and with PER2, as described below. For regulating circadian periods and/or metabolism by the ability to modulate CRY molecular structures, various chemicals have been created which bind to CRYs and which affect them. One such chemical is KL001 [28]. Reportedly, FBXL3 binds to CRY2 by occupying CRY2's binding pocket to FAD [29]. Also, KL001 inhibits FBXL3-dependent degradation of CRYs by competing with FAD for CRY binding, causing lengthening of the period of the molecular clock $[28,30]$. In terms of metabolism, the role of CRYs in the regulation of hepatic gluconeogenesis has been demonstrated. In addition to the effect on the circadian period, in primary hepatocytes, KL001 affects gluconeogenesis through action to CRYs [28]. CRY inhibits glucagon-stimulated cAMP production through regulating a Gs $\alpha$ subunit of a G protein [31]. SREBP1c regulates gluconeogenesis by CRY1-mediated FOXO1 degradation [32]. CRYs also regulate $\mathrm{NF} \kappa \mathrm{B}$ signalling by binding directly to adenylate cyclase and by limiting the production of cAMP [33]. CRYs bind directly to the glucocorticoid receptor and change the transcriptional response to glucocorticoids [34, 35]. The crystal structure of a CRY1-PER2 complex has been demonstrated by Schmalen et al. [36]. They presented new aspects of the molecular regulation of CRYs: zinc ion plays important roles in the formation of the CRY1-PER2 protein complex [36]. Also, cysteine414, which functions as an intermolecular zinc-binding site located in CRY1, plays a pivotal role in the binding of CRY1 to PER2, in conjunction with three other zinc-binding amino acid residues in both CRY1 and PER2 [36]. Another group determined the crystal structure of CRY2-PER2 complex [37] and showed that the relevant cysteine in CRY2 [38] also functions as an intermolecular zinc-binding site [37]. Details of the structure of CRYs based on recent structural analyses are discussed in a review [39].

\section{Unusual Circadian Rhythms and Diabetes Mellitus in C414A CRY1 Tg Mice}

The author previously generated transgenic mice ubiquitously overexpressing mouse CRY1 as well as mouse CRY1 having a mutation, cysteine 414 being replaced with alanine (C414A CRY1 Tg mice: previously designated as CRY1-AP Tg mice) [38]. Actually, C414A CRY1 severely reduces its binding ability to PER2 by in vitro examination [36]. The author generated two expression lines for C414A CRY1 Tg mice: high $(\mathrm{H})$ and low $(\mathrm{L})$ lines [38]. Fundamentally, they show a similar phenotype in terms of pathophysiological phenotypes [40]. Mice overexpressing intact CRY1 (CRY1 Tg mice) generated almost normal circadian rhythms in wheel-running activity [38]. In sharp contrast, the mice overexpressing the mutant CRY1 (hereinafter designated simply as Tg mice) displayed long free-running periods depending on the expression levels of transgenes [38]. The following refers only to the $\mathrm{H}$ line of $\mathrm{Tg}$ mice. They also showed abnormalities in both lightentrained [38] and food-entrained locomotor rhythms [15]. Detailed unique features of the SCN, which governs such unusual rhythms in $\mathrm{Tg}$ mice, are discussed in the literature [15]. In the Tg mice liver, the circadian expression of E-boxdriven genes, Per2 and Dbp, was depressed markedly [38], suggesting the hyperrepressive ability in C414A CRY1 to the E-box-driven clock gene and also to E-box-driven clockcontrolled genes in vivo. Results demonstrated that Crydeficient MEF cells show that C414A CRY1 actually depresses the amplitude of E-box-driven molecular clock and lengthens its period [37], which is inconsistent with the feature of $\mathrm{Tg}$ mice in terms of their extreme long free-running periods [38].

The author found that $\mathrm{Tg}$ mice show diabetes mellitus [38] in addition to unusual circadian behaviors [15, 38]. The features of diabetes are summarized as follows [40]. (1) Symptoms in Tg mice show sex-dependence: only male $\mathrm{Tg}$ mice show diabetes. (2) $\mathrm{Tg}$ mice at six weeks, the age at which blood glucose levels begin to elevate, already show glucose intolerance and exhibit no discernible insulin resistance. (3) At 9 weeks of age, neither hypercholesterolemia nor hypertriglyceridemia was observed in $\mathrm{Tg}$ mice. (4) Immunohistochemical analyses conducted at 19 and 40 weeks demonstrated that the insulin-stained area in the islet of $\mathrm{Tg}$ mice was smaller than that of wild-type controls. The extent of the decrease progressed age-dependently. (5) Levels of glucose-stimulated insulin secretion in $\mathrm{Tg}$ mice at 27 weeks were reduced compared with those of wildtype controls. Results suggest that, in Tg mice, the function of the insulin secretion of $\beta$-cell and the development of $\beta$-cell from a young age must be impaired to a certain degree. Consequently, Tg mice were expected to serve as an animal model of the insulin-secretory defect in human, such as maturity onset diabetes of the young (MODY [41]), a disease that is characterized by early onset and $\beta$-cell dysfunction [40]. At 40 weeks, male Tg mice showed higher serum triglyceride concentrations than wild-type controls did [38]. This phenomenon is attributable to the secondary effects of severe diabetes (diabetic lipemia) and does not contradict previously reported conclusions [40]. Actually, the blood glucose level increases age-dependently and reaches an extremely high level in male $\mathrm{Tg}$ mice $[38,40]$. Already at 40 weeks of age (ca. 10 months of age), Tg mice exhibit various severe symptoms of diabetes: a decrease in body weight accompanied by swelling out of lower abdomen and 
TABLE 1: Expression change in clock-related genes of isolated islets. The mice were kept in LD 14:10 as described [15]. The lighting period was 07:00-21:00, and islets were harvested during 11:00-13:00.

\begin{tabular}{lcc}
\hline Gene name & Fold change (Tg/wild-type) \\
\hline Hif & Down & 9.60 \\
Per3 & Down & 9.48 \\
Ror $\gamma$ & Down & 8.68 \\
Bmal1 & Up & 5.90 \\
Cryl (endogenous) & Down & 4.98 \\
Dbp & Down & 4.60 \\
Fbxl21 & Down & 4.55 \\
Tef & Down & 4.52 \\
Per2 & Down & 3.57 \\
Per1 & Down & 2.47 \\
Cry2 & Down & 2.33 \\
\hline
\end{tabular}

wet hindquarters caused by excessive polydipsia and polyuria [38, and unpublished data]. The author and colleagues have reported that lowered proliferation of $\beta$-cells is responsible for age-dependent $\beta$-cell loss in Tg mice [42]. The expression of transcription factors MAF-A and PDX-1, which are known to be important for the $\beta$-cell maturation and function, is reduced in the islet of Tg mice as they age [42]. The decreased expression of Insulin, Glucokinase, and Glut2 from a young age in the pancreas of $\mathrm{Tg}$ mice has also been reported [42]. In addition to the findings on C414A CRY1 Tg mice, the results of many intensive studies have implicated the involvement of clock genes in $\beta$-cell function [43-50]. The physiological consequences of various clock gene-modified animals are described in a review [51].

\section{Characteristic Features of Gene Expressions in the Islets of Young Tg Mice}

Recent DNA microarray analysis of islets derived from $\mathrm{Tg}$ mice at 4 weeks of age showed that the mRNA levels of Ebox-driven clock genes were markedly reduced in the islets of $\mathrm{Tg}$ mice (Table 1) [52], indicating malfunction of the molecular clock in the islets of Tg mice. Clock-related and metabolism-related transcription factors, DBP, RER-ERB $\alpha$, and $\operatorname{ROR} \gamma$, were reduced severely in the pancreas or islets of Tg mice $[42,52,53]$. Reportedly, these proteins are expressed in pancreatic $\beta$-cells. They can directly or indirectly affect the function of $\beta$-cells $[46,54-56]$. In line with the decreased proliferation of $\beta$-cells [42], the mRNA levels of various genes involved in cell-cycle control were altered [52]. Moreover, the expressions of various secretory proteins including inflammatory cytokines, chemokines, growth factors, and tissue remodelling factors were promoted in the islet of $\mathrm{Tg}$ mice [52]. This expression pattern is reminiscent of that of senescence-associated secretory phenotype (SASP) [57]. In silico analyses were conducted to trace the causes of this peculiar pattern of expression in the islet, based on DNA microarray data [53]. Results show that the overexpression of C414A CRY1 influences various signalling pathways in the islet. Particularly, aberrant activation by C414A CRY1 in the NF $\kappa \mathrm{B}$-mediated and also in the glucocorticoid receptor(GR-) mediated signalling pathways can play central roles in unusual features of $\beta$-cells in $\mathrm{Tg}$ mice [53]. In harmony with that, $\mathrm{NF} \kappa \mathrm{B}$ signalling is known to play important roles in the induction of SASP [57]. The involvement of CRYs in both GR-mediated and NFאB-mediated signalling pathways has already been reported as described above [3335]. Glucocorticoids influence $\beta$-cell function and also their population $[58,59]$. It is noteworthy that the mRNA and protein levels of RGS (regulator of G protein signalling) 4 increased markedly [53]. By inactivating $\mathrm{G} \alpha \mathrm{q}$, a subtype of $\mathrm{G}$ protein $\alpha$ subunits, it acts as a negative regulator of insulin release from pancreatic $\beta$-cells via $\mathrm{M}_{3}$ muscarinic acetylcholine receptor [60]. Disturbance in $\mathrm{G} \alpha \mathrm{q}$ signalling pathways can affect not only insulin secretion but also the proliferation of $\beta$-cells in mice [61]. Rgs16 is an E-box-driven clock gene [62]. RGS16 is a protein known to inhibit G $\alpha \mathrm{i} / \mathrm{o}$ [62]. Actually, the mRNA level of the $R g s 16$ was reduced in the islet of Tg mice [53], which is consistent with its reduced expression in the liver of $\mathrm{Tg}$ mice [15]. A recent report described that RGS16 regulates not only insulin secretion but also $\beta$-cell proliferation [63]. Consequently, disturbance in the signalling pathways mediated by RGS4 and RGS16 can cause not only insulin secretion but also the proliferation of $\beta$-cells in Tg mice. Collectively, in addition to the dysfunction of circadian clock in the islet, the combined influences of multiple pathways can cause the insulin-secretory defect and can cause severely decreased proliferation of $\beta$-cells in $\mathrm{Tg}$ mice from a young stage.

\section{Abnormality in the Islet Architecture of Tg Mice}

In $\mathrm{Tg}$ mice, the increase of the $\alpha$-cell $/ \beta$-cell ratio and gross abnormality in the distribution of $\alpha$-cells becomes conspicuous as they grow (Figure 3) [42]. The islet area per pancreas in Tg was smaller than that in wild-type controls [42]. To investigate the related mechanisms, $\alpha$-cell proliferation was measured using immunohistochemical analysis at 2 weeks of age. Results showed no significant difference in $\alpha$-cell proliferation between $\mathrm{Tg}$ and wild-type mice [53]. In addition, the glucagon-positive area per islet was increased from a young stage at 2 weeks [53] and 4 weeks [42]. Consequently, the unusual composition of cells is attributable in the literature mainly to "collapse of islets" [64] because of the specific loss of $\beta$-cells that mainly results from decreased proliferation in Tg mice.

\section{Unusual Ductal Structures in the Pancreas}

Immunohistochemical analyses have shown that unusually enlarged duct-like SOX9 positive structures (SOX9 is a transcription factor known as a ductal marker as well as a pancreatic endocrine progenitor marker) developed more conspicuously in pancreases in $\mathrm{Tg}$ mice than in those of wild-type controls [52, 53]. In $\mathrm{Tg}$ mice, this phenomenon became discernible histologically at the mature stage [52, 53]. Numerous duct-like cells were PCNA-positive [53], 


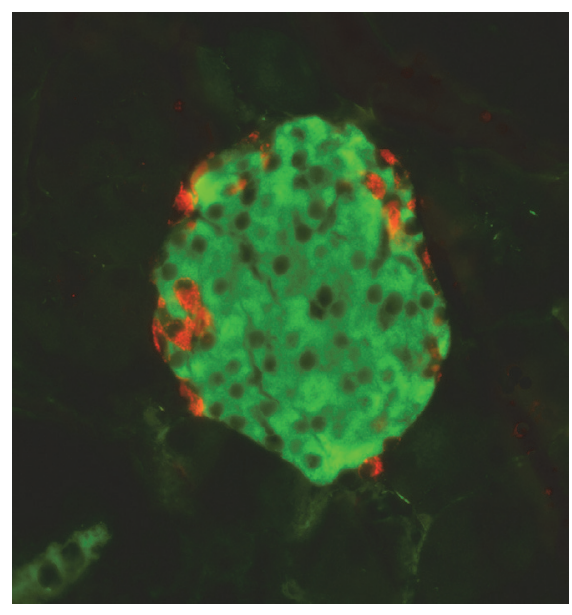

(a)

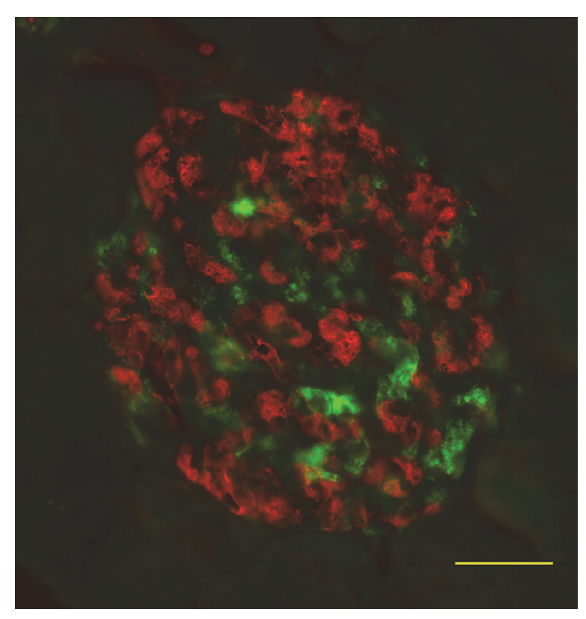

(b)

FIGURE 3: Coimmunostaining for insulin and glucagon of islets. Pancreas sections from wild-type (a) and Tg (b) mice at 40 weeks of age costained with antibodies to insulin (green) and glucagon (red). Bar, $40 \mu \mathrm{m}$.

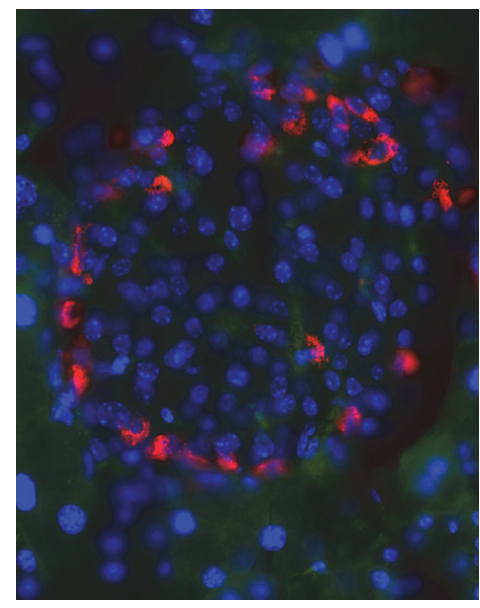

(a)

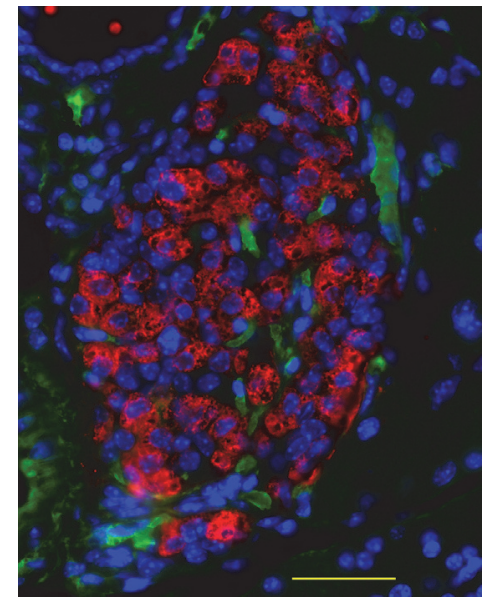

(b)

Figure 4: Increase in vascular formation in the islets of Tg mice. Pancreas sections from wild-type (a) and Tg (b) mice at 40 weeks of age costained with antibodies to PECAM1 (green) and glucagon (red) and counterstained with DAPI (blue) for nuclear staining. Bar, $40 \mu \mathrm{m}$.

suggesting that enhanced proliferation of the cells is involved in the development of the duct-like structures. At the mature stage, higher numbers of the vascular networks in the islets in Tg mice than those of wild-type mice were observed (Figure 4) [53]. Consistent with that observation, from the young stage, the mRNA level of Angiopoietin 2 was elevated in Tg mice islets [52]. It is noteworthy that previous reports have described that transgenic mice of the constitutively active form of constituents of insulin signalling pathway (Akt [65], Foxo1 [66]) showed similar expanded ductal structures, which contain proliferation marker-positive cells. Particularly, transgenic mice in which the overexpression of constitutively active form of FOXO1 in the pancreas uses $P d x-1$ promoter showed a similar phenotype to that of C414A CRY1 Tg mice: The FOXO1 Tg mice showed impaired glucose tolerance with the reduction in the $\beta$-cell mass accompanied by the reduction of MAF-A and PDX-1 [66]. In addition, the formation of hypervascular networks was observed in the islets of the FOXO1 Tg mice [66]. In this respect, a recent report describing that CRY1 is involved in the degradation of nuclear FOXO1 in conjunction with a ubiquitin ligase, MDM2 [32], is quite suggestive of the mechanism of unusual ductal structure growth. Possibly, C414A CRY1 inhibits dominant-negatively normal CRY1 for the degradation of FOXO1. Thereby, it functions as an activator of FOXO1 mediated signalling pathway in the $\mathrm{Tg}$ mice pancreas. Therefore, the overactivation of FOXO1 might be the primary cause of the induction of ductal structures in Tg mice. Moreover, deregulation of signalling cascades, such as Hedgehog-, Notch-, and Wnt-signalling pathways, might cause the development of the unusual ductal structures in Tg mice. C414A CRY1 might affect mouse Timeless (TIM; 


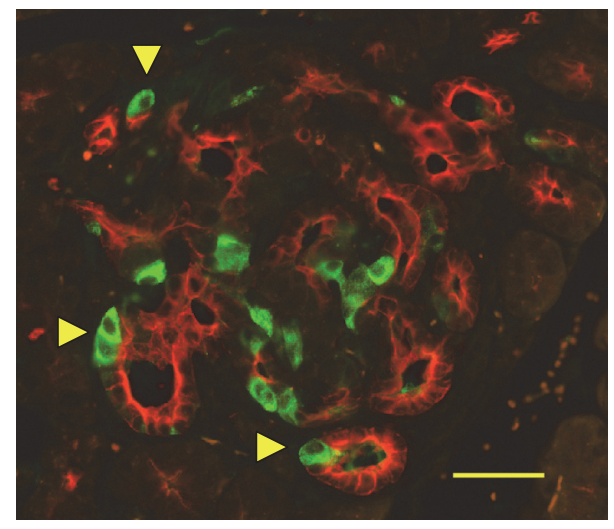

FIGURE 5: Insulin-expressing cells in the Tg mice ductal structures. Pancreas sections from $\mathrm{Tg}$ mice at 40 weeks of age costained with antibodies to insulin (green) and cytokeratin 17/19 (red). Yellow triangles denote insulin-positive cells in the structure. Bar, $40 \mu \mathrm{m}$.

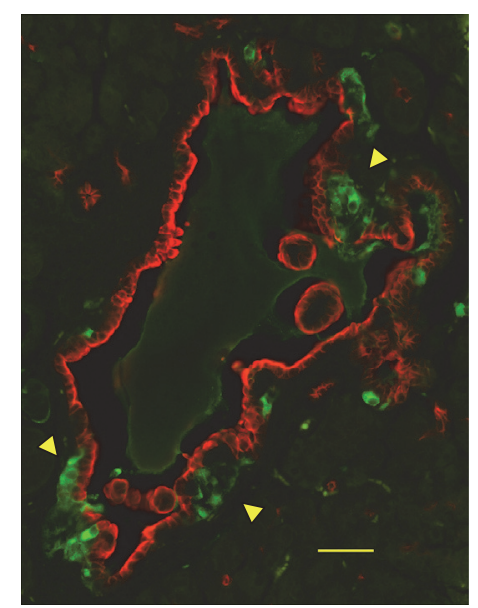

FIgURE 6: Clusters of insulin-positive cells located near the ductlike structure in Tg mice. Pancreas sections from Tg mice at 40 weeks of age were costained with antibodies to insulin (green) and cytokeratin 17/19 (red). Bar, $40 \mu \mathrm{m}$.

TIM is known as a CRY-binding protein [39]) and might perturb the function of TIM in the pancreas, possibly leading to aberrant activation of the signalling pathways, as described in one report [67]. Actually, some constituents of Hedgehogsignalling and Wnt-signalling pathways were elevated in the Tg mice islet [52]. Recently, it was proposed that TIM plays a role in DNA damage response in conjunction with PARP1 [68]. Perturbation of TIM by C414A CRY1 might cause the accumulation of DNA damage, which can contribute to the lowered proliferation of $\beta$-cells in Tg mice. The duct-like structures contained insulin-positive cells (Figure 5) [53]. In addition, the clusters of insulin-positive cells were located near the duct-like structure (Figure 6) [53]. These results strongly suggest that the neogenesis of $\beta$-cells occurs there. One review has summarized experimentally derived models of $\beta$-cell neogenesis reported to date and the roles of ductal cells in respect of the source of $\beta$-cells [69]. At the mature stage, infiltrated macrophage cells were observed frequently in the islet of $\mathrm{Tg}$ mice [53]. Chemokine/cytokine-induced epithelial-mesenchymal transition (EMT) in the process of $\beta$-cell neogenesis from ductal cells was proposed recently [70]. A similar induction mechanism to that of EMT is likely to be involved, at least partially, in generating new $\beta$-cells from duct-like structures, in which the secreted cytokines and chemokines play relevant roles in $\mathrm{Tg}$ mice because of SASPlike characters of $\beta$-cells [52] and by recruited macrophage cells to the islet [53]. Taken together, remodelling in the vascular network in the islet progresses with age. Infiltrated macrophages might be involved, at least partially, in the processes.

\section{Future Directions}

Regarding cross-talk between the circadian clock and the regulation of functions of $\beta$-cells, the elucidation of the circadian-hormonal effects is expected to become increasingly important. This trend is exemplified by recent reports: melatonin, a hormone that exhibits some relation to circadian rhythm [71], directly regulates insulin secretion of $\beta$ cells [72]. Glucocorticoids, the concentrations of which also fluctuate in a circadian manner [71], influence $\beta$-cell function as discussed above. Moreover, it has been demonstrated that thyroid hormone $\mathrm{T}_{3}$ stimulates $\beta$-cell maturation by increasing MAF-A expression [73]. Circadian control of thyroid hormones is described in a review [74]. Consequently, appropriate timing of the daily exposure of these hormones to $\beta$ cells is expected to be important not only for the fine-tuning of $\beta$-cell functions, but also for the maintenance of $\beta$-cells.

The gross abnormality displayed remarkably in the islet and pancreas in Tg mice demonstrates that CRY functions in mammals are limited not only to the part of TTFL of circadian clock; they also strongly affect $\beta$-cell proliferation and physiology. Particularly, it is intriguing to imagine that the Zn-binding of CRY-PER complex might have some additional uncovered roles in the $\beta$-cell function because contents and cellular levels of zinc in $\beta$-cells are known to be exceptionally high compared with those of other cells [75]. To enhance our understanding of why ubiquitous overexpression of C414A CRY1 especially affects $\beta$-cells in mice, further experiments must be conducted.

\section{Competing Interests}

The author declares that there are no competing interests.

\section{Acknowledgments}

The author would like to thank Professors M. Gannon (Vanderbilt University) and S. Bonner-Weir (Joslin Diabetes Center) for valuable discussions related to the islet architecture. Special appreciation is extended to Professors H. Mizukami (Hirosaki University), S. Yagihashi (Hirosaki University), and W. Nishimura (Jichi Medical University) for technical advice related to islet isolation and analysis of the $\beta$-cell. Work related to C414A CRY1 Tg mice was supported by Akio Yuki plan 2008 (Yamagata University; Grant no. 35), JSPS KAKENHI [Grant nos. 16790142, 18790168, 21590429, 
24590473, and 15K08417], and the Joint Research Program of Joint Usage/Research Center at the Institute of Development, Aging and Cancer (IDAC), Tohoku University (Grant nos. 30 [2013], 6 [2014], and 3 [2015]).

\section{References}

[1] A. R. Cashmore, J. A. Jarillo, Y.-J. Wu, and D. Liu, "Cryptochromes: blue light receptors for plants and animals," Science, vol. 284, no. 5415, pp. 760-765, 1999.

[2] S. Okano, S.-I. Kanno, M. Takao et al., "A putative blue-light receptor from Drosophila melanogaster," Photochemistry and Photobiology, vol. 69, no. 1, pp. 108-113, 1999.

[3] A. Sancar, "Mechanisms of DNA repair by photolyase and excision nuclease (Nobel Lecture)," Angewandte Chemie International Edition, vol. 55, no. 30, pp. 8502-8527, 2016.

[4] J. D. Alvarez, "Genetic basis for circadian rhythms in mammals," in Molecular Biology of Circadian Rhythms, A. Sehgal, Ed., pp. 93-140, John Wiley \& Sons, New Jersey, NJ, USA, 2004.

[5] F. K. Stephan and I. Zucker, "Circadian rhythms in drinking behavior and locomotor activity of rats are eliminated by hypothalamic lesions," Proceedings of the National Academy of Sciences of the United States of America, vol. 69, no. 6, pp. 15831586, 1972.

[6] R. Y. Moore and V. B. Eichler, "Loss of a circadian adrenal corticosterone rhythm following suprachiasmatic lesions in the rat," Brain Research, vol. 42, no. 1, pp. 201-206, 1972.

[7] S. T. Inouye and H. Kawamura, "Persistence of circadian rhythmicity in a mammalian hypothalamic 'island' containing the suprachiasmatic nucleus," Proceedings of the National Academy of Sciences of the United States of America, vol. 76, no. 11, pp. 5962-5966, 1979.

[8] P. J. Van Der Spek, K. Kobayashi, D. Bootsma, M. Takao, A. P. M. Eker, and A. Yasui, "Cloning, tissue expression, and mapping of a human photolyase homolog with similarity to plant blue-light receptors," Genomics, vol. 37, no. 2, pp. 177-182, 1996.

[9] T. Todo, H. Ryo, K. Yamamoto et al., "Similarity among the Drosophila (6-4)photolyase, a human photolyase homolog, and the DNA photolyase-blue-light photoreceptor family," Science, vol. 272, no. 5258, pp. 109-112, 1996.

[10] D. S. Hsu, X. Zhao, S. Zhao et al., "Putative human bluelight photoreceptors hCRY1 and hCRY2 are flavoproteins," Biochemistry, vol. 35, no. 44, pp. 13871-13877, 1996.

[11] K. Kobayashi, S. Kanno, B. Smit, G. T. van der, M. Horst, and A. Yasui, "Characterization of photolyase/blue-light receptor homologs in mouse and human cells," Nucleic Acids Research, vol. 26, no. 22, pp. 5086-5092, 1998.

[12] G. T. J. Van Der Horst, M. Muijtjens, K. Kobayashi et al., "Mammalian Cryl and Cry2 are essential for maintenance of circadian rhythms," Nature, vol. 398, no. 6728, pp. 627-630, 1999.

[13] M. H. Vitaterna, C. P. Selby, T. Todo et al., "Differential regulation of mammalian period genes and circadian rhythmicity by cryptochromes 1 and 2," Proceedings of the National Academy of Sciences of the United States of America, vol. 96, no. 21, pp. 1211412119, 1999.

[14] K. Kume, M. J. Zylka, S. Sriram et al., "mCRY1 and mCRY2 are essential components of the negative limb of the circadian clock feedback loop," Cell, vol. 98, no. 2, pp. 193-205, 1999.

[15] S. Okano, A. Yasui, K. Hayasaka, and O. Nakajima, "Unique food-entrained circadian rhythm in cysteine414-alanine mutant mCRY1 transgenic mice," Sleep and Biological Rhythms, vol. 14, no. 3, pp. 261-269, 2016.

[16] P. Emery and S. M. Reppert, "A rhythmic ror," Neuron, vol. 43, no. 4, pp. 443-446, 2004.

[17] J. L. Price, "Drosophila melanogaster: a model system for molecular chronobiology," in Molecular Biology of Circadian Rhythms, A. Sehgal, Ed., pp. 93-140, John Wiley \& Sons, New Jersey, NJ, USA, 2004.

[18] L. J. Ashmore and A. Sehgal, "A fly's eye view of circadian entrainment," Journal of Biological Rhythms, vol. 18, no. 3, pp. 206-216, 2003.

[19] H. G. Hiscock, S. Worster, D. R. Kattnig et al., "The quantum needle of the avian magnetic compass," Proceedings of the National Academy of Sciences, vol. 113, no. 17, pp. 4634-4639, 2016.

[20] K. A. Lamia, U. M. Sachdeva, L. Di Tacchio et al., "AMPK regulates the circadian clock by cryptochrome phosphorylation and degradation," Science, vol. 326, no. 5951, pp. 437-440, 2009.

[21] S. M. Siepka, S.-H. Yoo, J. Park et al., "Circadian mutant overtime reveals F-box protein FBXL3 regulation of cryptochrome and period gene expression," Cell, vol. 129, no. 5, pp. 1011-1023, 2007.

[22] S. I. H. Godinho, E. S. Maywood, L. Shaw et al., "The after-hours mutant reveals a role for Fbxl3 in determining mammalian circadian period," Science, vol. 316, no. 5826, pp. 897-900, 2007.

[23] L. Busino, F. Bassermann, A. Maiolica et al., "SCF ${ }^{F b x l 3}$ controls the oscillation of the circadian clock by directing the degradation of cryptochrome proteins," Science, vol. 316, no. 5826, pp. 900-904, 2007.

[24] A. Hirano, K. Yumimoto, R. Tsunematsu et al., "FBXL21 regulates oscillation of the circadian clock through ubiquitination and stabilization of cryptochromes," Cell, vol. 152, no. 5, pp. 1106-1118, 2013.

[25] S.-H. Yoo, J. A. Mohawk, S. M. Siepka et al., "Competing E3 ubiquitin ligases govern circadian periodicity by degradation of CRY in nucleus and cytoplasm," Cell, vol. 152, no. 5, pp. 10911105, 2013.

[26] A. Hirano, T. Nakagawa, H. Yoshitane et al., "USP7 and TDP-43: pleiotropic regulation of cryptochrome protein stability paces the oscillation of the mammalian circadian clock," PLOS ONE, vol. 11, no. 4, Article ID e0154263, 2016.

[27] I. Lim, Y. Jung, D. Kim, K. Kim, and N. S. Foulkes, "HnRNP $\mathrm{Q}$ has a suppressive role in the translation of mouse cryptochromel," PLOS ONE, vol. 11, no. 7, Article ID e0159018, 2016.

[28] T. Hirota, J. W. Lee, P. C. St John et al., "Identification of small molecule activators of cryptochrome," Proceedings of the National Academy of Sciences of the United States of America, vol. 111, no. 5, pp. 2040-2045, 2014.

[29] W. Xing, L. Busino, T. R. Hinds et al., " $S C F{ }^{F B X L 3}$ ubiquitin ligase targets cryptochromes at their cofactor pocket," Nature, vol. 496, no. 7443, pp. 64-68, 2013.

[30] S. Nangle, W. Xing, and N. Zheng, "Crystal structure of mammalian cryptochrome in complex with a small molecule competitor of its ubiquitin ligase," Cell Research, vol. 23, no. 12, pp. 1417-1419, 2013.

[31] E. E. Zhang, Y. Liu, R. Dentin et al., "Cryptochrome mediates circadian regulation of cAMP signaling and hepatic gluconeogenesis," Nature Medicine, vol. 16, no. 10, pp. 1152-1156, 2010.

[32] H. Jang, G. Y. Lee, C. P. Selby et al., "SREBP1c-CRY1 signalling represses hepatic glucose production by promoting FOXO1 degradation during refeeding," Nature Communications, vol. 7, Article ID 12180, 2016. 
[33] R. Narasimamurthy, M. Hatori, S. K. Nayak, F. Liu, S. Panda, and I. M. Verma, "Circadian clock protein cryptochrome regulates the expression of proinflammatory cytokines," Proceedings of the National Academy of Sciences of the United States of America, vol. 109, no. 31, pp. 12662-12667, 2012.

[34] K. A. Lamia, S. J. Papp, R. T. Yu et al., "Cryptochromes mediate rhythmic repression of the glucocorticoid receptor," Nature, vol. 480, no. 7378, pp. 552-556, 2011.

[35] N. Koike, S.-H. Yoo, H.-C. Huang et al., "Transcriptional architecture and chromatin landscape of the core circadian clock in mammals," Science, vol. 338, no. 6105, pp. 349-354, 2012.

[36] I. Schmalen, S. Reischl, T. Wallach et al., "Interaction of circadian clock proteins CRY1 and PER2 is modulated by zinc binding and disulfide bond formation," Cell, vol. 157, no. 5, pp. 1203-1215, 2014.

[37] S. N. Nangle, C. Rosensweig, N. Koike et al., "Molecular assembly of the period-cryptochrome circadian transcriptional repressor complex," eLife, vol. 3, p. e03674, 2014.

[38] S. Okano, M. Akashi, K. Hayasaka, and O. Nakajima, "Unusual circadian locomotor activity and pathophysiology in mutant CRY1 transgenic mice," Neuroscience Letters, vol. 451, no. 3, pp. 246-251, 2009.

[39] T. Merbitz-Zahradnik and E. Wolf, "How is the inner circadian clock controlled by interactive clock proteins?: structural analysis of clock proteins elucidates their physiological role," FEBS Letters, vol. 589, no. 14, Article ID 37180, pp. 1516-1529, 2015.

[40] S. Okano, K. Hayasaka, M. Igarashi, H. Iwai, Y. Togashi, and O. Nakajima, "Non-obese early onset diabetes mellitus in mutant cryptochromel transgenic mice," European Journal of Clinical Investigation, vol. 40, no. 11, pp. 1011-1017, 2010.

[41] S. S. Fajans and G. I. Bell, "MODY: history, genetics, pathophysiology, and clinical decision making," Diabetes Care, vol. 34, no. 8, pp. 1878-1884, 2011.

[42] S. Okano, K. Hayasaka, M. Igarashi, Y. Togashi, and O. Nakajima, "Characterization of age-associated alterations of islet function and structure in diabetic mutant cryptochrome 1 transgenic mice," Journal of Diabetes Investigation, vol. 4, no. 5, pp. 428-435, 2013.

[43] B. Marcheva, K. M. Ramsey, E. D. Buhr et al., "Disruption of the clock components CLOCK and BMAL1 leads to hypoinsulinaemia and diabetes," Nature, vol. 466, no. 7306, pp. 627-631, 2010.

[44] L. A. Sadacca, K. A. Lamia, A. S. DeLemos, B. Blum, and C. J. Weitz, "An intrinsic circadian clock of the pancreas is required for normal insulin release and glucose homeostasis in mice," Diabetologia, vol. 54, no. 1, pp. 120-124, 2011.

[45] J. Lee, M.-S. Kim, R. Li et al., "Loss of Bmall leads to uncoupling and impaired glucose-stimulated insulin secretion in $\beta$-cells," Islets, vol. 3, no. 6, pp. 381-388, 2011.

[46] E. Vieira, L. Marroquí, T. M. Batista et al., "The clock gene Reverb $\alpha$ regulates pancreatic $\beta$-cell function: modulation by leptin and high-fat diet," Endocrinology, vol. 153, no. 2, pp. 592-601, 2012.

[47] J. Lee, M. Moulik, Z. Fang et al., "Bmall and $\beta$-cell clock are required for adaptation to circadian disruption, and their loss of function leads to oxidative stress-induced $\beta$-cell failure in mice," Molecular and Cellular Biology, vol. 33, no. 11, pp. 2327-2338, 2013.

[48] P. Pulimeno, T. Mannic, D. Sage et al., "Autonomous and selfsustained circadian oscillators displayed in human islet cells," Diabetologia, vol. 56, no. 3, pp. 497-507, 2013.
[49] J. Qian, G. D. Block, C. S. Colwell, and A. V. Matveyenko, "Consequences of exposure to light at night on the pancreatic islet circadian clock and function in rats," Diabetes, vol. 62, no. 10, pp. 3469-3478, 2013.

[50] M. Perelis, B. Marcheva, K. M. Ramsey et al., "Pancreatic $\beta$ cell enhancers regulate rhythmic transcription of genes controlling insulin secretion," Science, vol. 350, no. 6261, 2015.

[51] K. Eckel-Mahan and P. Sassone-Corsi, "Metabolism and the circadian clock converge," Physiological Reviews, vol. 93, no. 1, pp. 107-135, 2013.

[52] S. Okano, A. Yasui, K. Hayasaka, M. Igarashi, and O. Nakajima, "Senescence-like phenotype in pancreatic $\beta$-cells in diabetic mutant cryptochromel transgenic mice (2299-P)," Diabetes, vol. 64, supplement 1, pp. A584-A585, 2015, https://ada.scientificposters.com/epsWelcome.cfm?CFID=5374608\&CFTOKEN= 640ba39c397alc08-74A9E72F-B30B-7210-AFE4033F4F63F33E.

[53] S. Okano, A. Yasui, S. Kanno, K. Hayasaka, M. Igarashi, and O. Nakajima, "Anomalous ductal structure in the islet of diabetic cysteine414-alanine-mCRY1 transgenic mice (2073-P)," Diabetes, vol. 65, supplement 1, p. A530, 2016, https://ada.scientificposters.com/epsWelcome.cfm?CFID=5374608\&CFTOKEN= 640ba39c397alc08-74A9E72F-B30B-7210-AFE4033F4F63F33E.

[54] N. Allaman-Pillet, R. Roduit, A. Oberson et al., "Circadian regulation of islet genes involved in insulin production and secretion," Molecular and Cellular Endocrinology, vol. 226, no. 1-2, pp. 59-66, 2004.

[55] H. Nakabayashi, Y. Ohta, M. Yamamoto et al., "Clock-controlled output gene Dbp is a regulator of Arnt/Hif-1 $\beta$ gene expression in pancreatic islet $\beta$-cells," Biochemical and Biophysical Research Communications, vol. 434, no. 2, pp. 370-375, 2013.

[56] E. Mühlbauer, I. Bazwinsky-Wutschke, S. Wolgast, K. Labucay, and E. Peschke, "Differential and day-time dependent expression of nuclear receptors $\operatorname{ROR} \alpha, \operatorname{ROR} \beta, \operatorname{ROR} \gamma$ and $\mathrm{RXR} \alpha$ in the rodent pancreas and islet," Molecular and Cellular Endocrinology, vol. 365, no. 2, pp. 129-138, 2013.

[57] A. Salminen, A. Kauppinen, and K. Kaarniranta, "Emerging role of NF- $\kappa \mathrm{B}$ signaling in the induction of senescence-associated secretory phenotype (SASP)," Cellular Signalling, vol. 24, no. 4, pp. 835-845, 2012.

[58] A. Rafacho, H. Ortsäter, A. Nadal, and I. Quesada, "Glucocorticoid treatment and endocrine pancreas function: implications for glucose homeostasis, insulin resistance and diabetes," Journal of Endocrinology, vol. 223, no. 3, pp. R49-R62, 2014.

[59] E. Gesina, F. Tronche, P. Herrera et al., "Dissecting the role of glucocorticoids on pancreas development," Diabetes, vol. 53, no. 9, pp. 2322-2329, 2004.

[60] I. R. de Azua, M. Scarselli, E. Rosemond et al., "RGS4 is a negative regulator of insulin release from pancreatic $\beta$-cells in vitro and in vivo," Proceedings of the National Academy of Sciences of the United States of America, vol. 107, no. 17, pp. 7999$8004,2010$.

[61] S. Jain, I. R. De Azua, H. Lu, M. F. White, J.-M. Guettier, and J. Wess, "Chronic activation of a designer $\mathrm{G}_{q}$-coupled receptor improves $\beta$ cell function," Journal of Clinical Investigation, vol. 123, no. 4, pp. 1750-1762, 2013.

[62] M. Doi, A. Ishida, A. Miyake et al., "Circadian regulation of intracellular G-protein signalling mediates intercellular synchrony and rhythmicity in the suprachiasmatic nucleus," Nature Communications, vol. 2, no. 1, article no. 327, 2011.

[63] K. Vivot, V. S. Moullé, B. Zarrouki et al., "The regulator of G-protein signaling RGS16 promotes insulin secretion and 
$\beta$-cell proliferation in rodent and human islets," Molecular Metabolism, vol. 5, no. 10, pp. 988-996, 2016.

[64] M. Kharouta, K. Miller, A. Kim et al., "No mantle formation in rodent islets-the prototype of islet revisited," Diabetes Research and Clinical Practice, vol. 85, no. 3, pp. 252-257, 2009.

[65] L. Elghazi, A. J. Weiss, D. J. Barker et al., "Regulation of pancreas plasticity and malignant transformation by Akt signaling," Gastroenterology, vol. 136, no. 3, pp. 1091.e8-1103.e8, 2009.

[66] O. Kikuchi, M. Kobayashi, K. Amano et al., "FoxO1 gain of function in the pancreas causes glucose intolerance, polycystic pancreas, and islet hypervascularization," PLOS ONE, vol. 7, no. 2, Article ID e32249, 2012.

[67] Z. Li, L. Ruan, S. Lin, and G. K. Gittes, "Clock controls timing of mouse pancreatic differentiation through regulation of Wntand Notch-based and cell division components," Biochemical and Biophysical Research Communications, vol. 359, no. 3, pp. 491-496, 2007.

[68] L. M. Young, A. Marzio, P. Perez-Duran et al., "TIMELESS forms a complex with PARP1 distinct from its complex with TIPIN and plays a role in the DNA damage response," Cell Reports, vol. 13, no. 3, pp. 451-459, 2015.

[69] S. Bonner-Weir, L. Guo, W.-C. Li et al., "Islet neogenesis: a possible pathway for beta-cell replenishment," Review of Diabetic Studies, vol. 9, no. 4, pp. 407-416, 2012.

[70] I. A. Valdez, E. Dirice, M. K. Gupta, J. Shirakawa, A. K. Teo, and R. Kulkarni, "Proinflammatory cytokines induce endocrine differentiation in pancreatic ductal cells via STAT3-dependent NGN3 activation," Cell Reports, vol. 15, no. 3, pp. 460-470, 2016.

[71] P. McNamara, "Hormonal rhythms," in Molecular Biology of Circadian Rhythms, A. Sehgal, Ed., pp. 231-253, John Wiley \& Sons, New Jersey, NJ, USA, 2004.

[72] T. Tuomi, C. Nagorny, P. Singh et al., "Increased melatonin signaling is a risk factor for type 2 diabetes," Cell Metabolism, vol. 23, no. 6, pp. 1067-1077, 2016.

[73] C. Aguayo-Mazzucato, A. Dilenno, J. Hollister-Lock et al., "MAFA and T3 drive maturation of both fetal human islets and insulin-producing cells differentiated from hESC," Journal of Clinical Endocrinology and Metabolism, vol. 100, no. 10, pp. 3651-3659, 2015.

[74] J. Philippe and C. Dibner, "Thyroid circadian timing: roles in physiology and thyroid malignancies," Journal of Biological Rhythms, vol. 30, no. 2, pp. 76-83, 2015.

[75] H. W. Davidson, J. M. Wenzlau, and R. M. O’Brien, "Zinc transporter 8 (ZnT8) and $\beta$ cell function," Trends in Endocrinology and Metabolism, vol. 25, no. 8, pp. 415-424, 2014. 


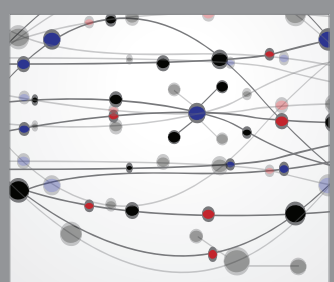

The Scientific World Journal
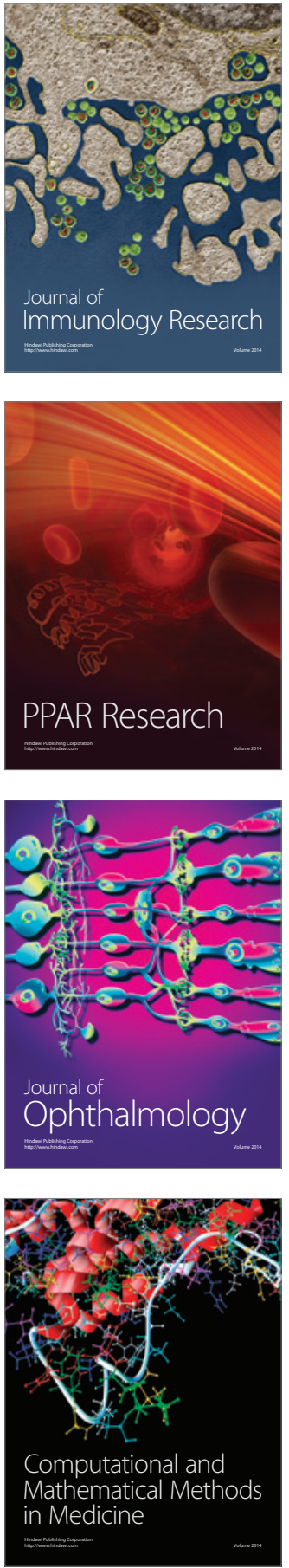

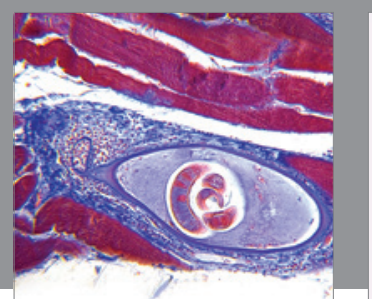

Gastroenterology Research and Practice

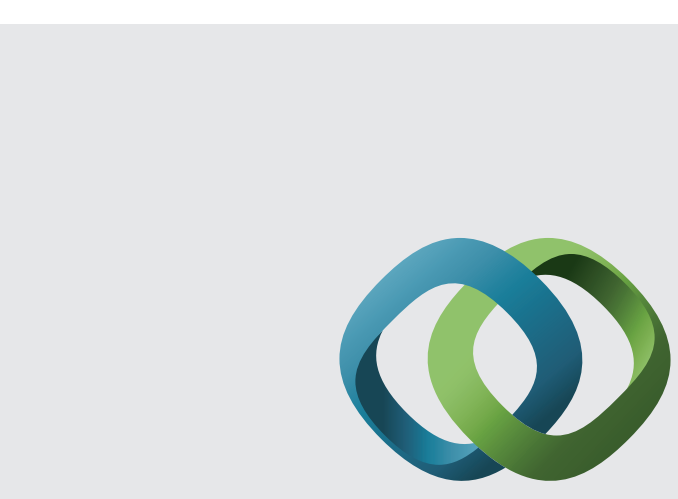

\section{Hindawi}

Submit your manuscripts at

http://www.hindawi.com
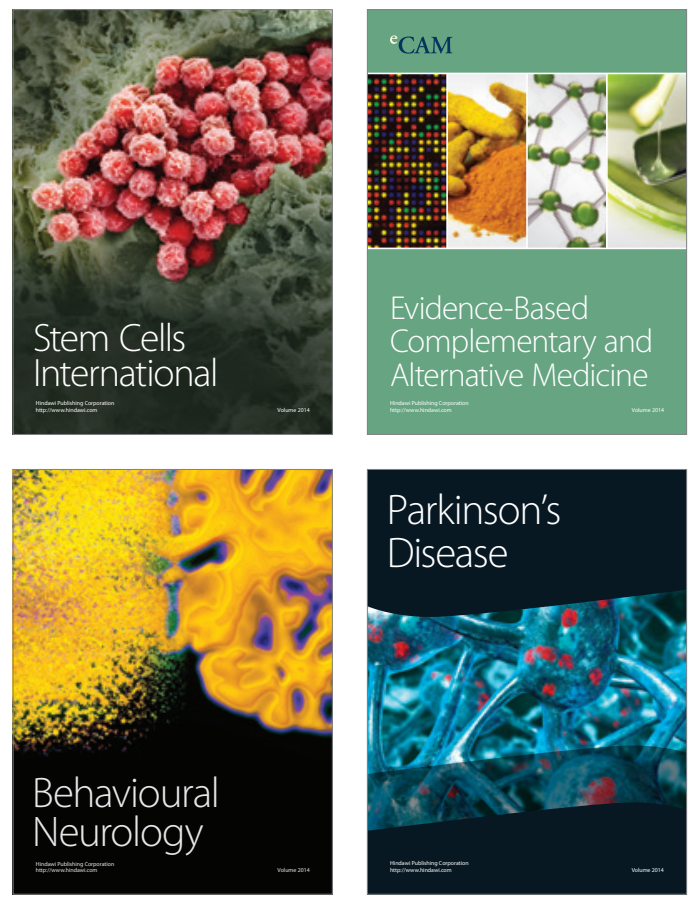
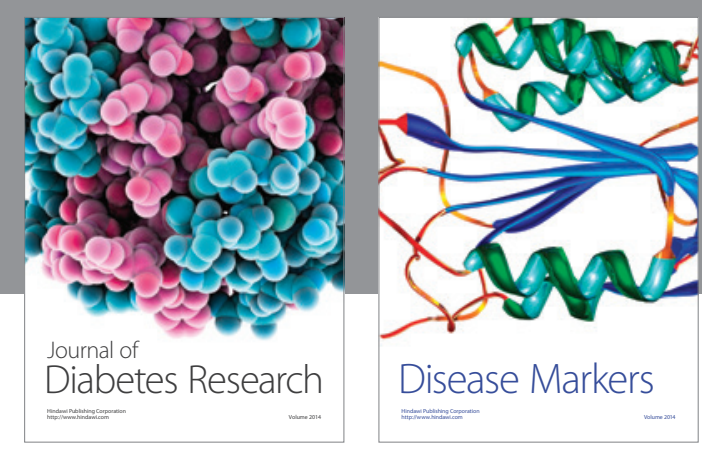

Disease Markers
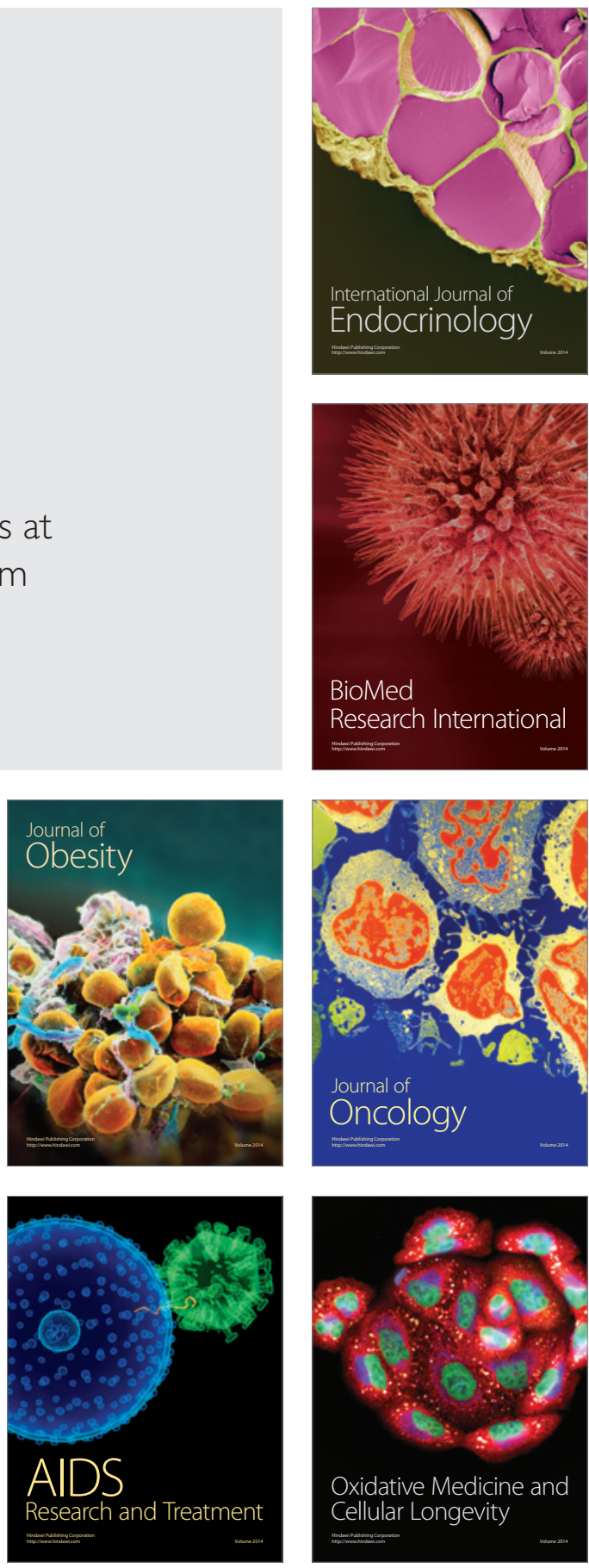